\title{
The importance of VRI and AOR segmentations in optical coherence tomography angiography
}

\begin{abstract}
In this editorial, it was aimed to call attention to the importance of the screening and the evaluation of the segmentations of vitreoretinal interface and avascular outer retina in optical coherence tomography angiography for subclinical or early pre-retinal and choroidal neovascularization. In optical coherence tomography angiography of a healthy subject, both segmentations of vitreoretinal interface and avascular outer retina do not show obvious vascular structure. Thus, these two segmentations can be used for the screening of preretinal and choroidal neovascularization in various ischemic vascular diseases.
\end{abstract}

Keywords: avascular outer retina, choroidal neovascularization, optical coherence tomography angiography, vitreo-retinal interface, pre-retinal neovascularization, screening
Volume 6 Issue 6 - 2017

Burak Turgut

Department of Ophthalmology, Firat University, Turkey

Correspondence: Burak Turgut, Associate Professor of Ophthalmology, Firat University, Faculty of Medicine, Department of Ophthalmology, 231 I 9 Elazig, Turkey, Tel +904242333555,

Email burakturgut@firat.edu.tr, drburakturgut@gmail.com

Received: April 21, 2017 | Published: April 24, 2017
Abbreviations: OCTA, optical coherence tomography angiography; VRI, vitreoretinal interface; AOR, avascular outer retina; PNV, preretinal neovascularization; OPL, outer plexiform layer; $\mathrm{CNV}$, choroidal neovascularization; RPE, sub-retinal pigment epithelium

\section{Editorial}

Optical coherence tomography angiography (OCTA) is a very useful angiography type for the evaluation of both retinal and choroidal vasculature. OCTA is noninvasive imaging method without adverse effects because the usage of a contrast agent is not needed in this imaging method. Using by OCTA, the vasculature and blood flow of retina and choroid can be visualized in detail through detecting motion contrast of erythrocytes in the vessel, using serial optical coherence tomography B-scans. OCTA has an ability to perform the separate segmentations including the vitreoretinal interface (VRI), the superficial retinal layer, the deep retinal layer, avascular outer retina (AOR), the choriocapillaris and the choroid (Figure 1). ${ }^{1-8}$ In a healthy eye, the VRI has no vascular structure though the ganglion cell layer of the retina has the superficial retinal capillary plexus based on vitreo-retinal histology and VRI segmentation of OCTA (Figure 1) VRI. ${ }^{1-8}$ Preretinal neovascularization (PNV) is characterized by networks of vessels lying on the surface of the retina and invading the cortical vitreous gel and extending into the vitreous cavity. PNV usually occurs in the ischemic retinal vascular diseases such as proliferative diabetic retinopathy, retinal vein occlusion, retinopathy of prematurity and sickle-cell disease retinopathy. ${ }^{9-13} \mathrm{PNV}$ in earlier stages of the pathogenesis of abnormal neovascularization event may be overlooked. VRI segmentation of OCTA can be used to screen and detect the early or subclinical PNV in these diseases. ${ }^{9-12}$ In the other hand, both the histological examinations and OCTA reports of retina in healthy subjects demonstrated that there is an avascular retinal region (avascular outer retina, AOR) in the deep or outer layer of the retina. AOR is the layer between the outer plexiform layer (OPL) in the inner border and ellipsoid zone in the outer border. The outer retina segmentation between the OPL and Bruch membrane by OCTA show no vascular blood flow in a normal eye (Figure 1) Avascular. ${ }^{1-8}$ Choroidal neovascularization (CNV) defines the development of the new blood vessels derived the choroid and the invasion into sub- retinal pigment epithelium (RPE) or subretinal space via a break in Bruch membrane. Type 1 choroidal neovascularization $(\mathrm{CNV})$ is characterized by vessels proliferating under the RPE and it is found in the subretinal space. Type $1 \mathrm{CNV}$ is also termed occult $\mathrm{CNV}$ because it can be poorly detected with dye-assisted angiography. In the OCT scans Type $1 \mathrm{CNV}$ is observed as localized fusiform serous or fibro-vascular RPE detachment. Additionally, polypoidal choroidal vasculopathy is also considered as a variation of this type CNV. ${ }^{14,15} \mathrm{In}$ newly studies, it has been reported that OCTA provides more precisely and comprehensively describe of Type 1 CNV in AMD [16-19]. Thus, OCTA can also be used for early detection of an occult CNV (Type 1 $\mathrm{CNV}$ ) in this AOR in the eyes with asymptomatic eyes in the stages of early or intermediate AMD.

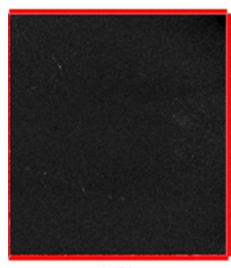

VRI

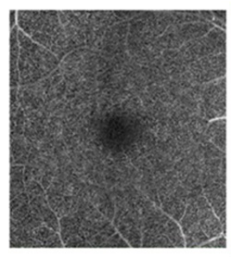

Deep

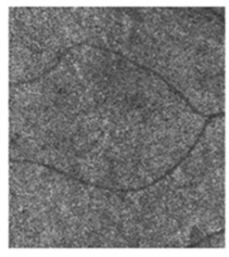

Choriocapillaris

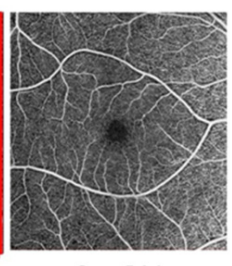

Superficial

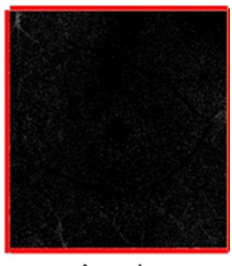

Avascular

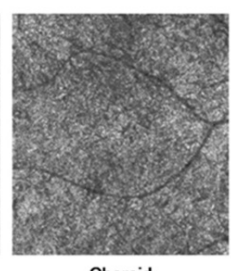

Choroid
Figure I The segmentations in optical coherence tomography angiography. The images with red frames belong to the segmentations of vitreoretinal interface (VRI) and avascular outer retina. 


\section{Conclusion}

The segmentations of VRI and AOR in OCTA have no any vascular neoformation and that of the observation in OCTA of a vascular structure in the segmentations of these segmentations may provide early detection any preretinal or choroidal NV, respectively.

\section{Acknowledgments}

None.

\section{Conflicts of interest}

The authors declare there are no conflicts of interest.

\section{References}

1. Jia Y, Bailey ST, Hwang ST, et al. Quantitative optical coherence tomography angiography of vascular abnormalities in the living human eye. Proc Natl Acad Sci U S A. 2015;112(18):E2395-E2402.

2. Turgut. Optical coherence tomography angiography-A general view. European Ophthalmic Review. 2016;10(1):39-42.

3. Gao SS, Jia Y, Zhang M, et al. Optical Coherence Tomography Angiography. Invest Ophthalmol Vis Sci. 2016;57(9): OCT27-OCT36.

4. Spaide RF, Klancnik JM, Cooney MJ. Retinal vascular layers imaged by fluorescein angiography and optical coherence tomography angiography. JAMA Ophthalmol. 2015;133(1):45-50.

5. Choi W, Mohler KJ, Potsaid B, et al. Choriocapillaris and choroidal microvasculature imaging with ultrahigh speed OCT angiography. PLoS ONE. 2013;8(12):e.81499.

6. Zhang Q, Huang Y, Zhang T, et al. Wide-field imaging of retinal vasculature using optical coherence tomography-based microangiography. J Biomed Opt. 2015;0(6):066008

7. Matsunaga D, Yi J, Puliafito C, et al. OCT angiography in healthy human subjects. Ophthalmic Surg Lasers Imaging Retina. 2014;5(6):510-515.

8. Wylegała A, Teper S, Dobrowolski D, et al. Optical coherence angiography: A review. Medicine. 2016;95(41): e4907.
9. De Carlo TE, Bonini Filho MA, Baumal CR, et al. Evaluation of Preretinal Neovascularization in Proliferative Diabetic Retinopathy Using Optical Coherence Tomography Angiography. Ophthalmic Surg Lasers Imaging Retina. 2016;47(2):115-119.

10. De Barros Garcia JMB, Isaac DLC, Avila M. Diabetic retinopathy and OCT angiography: clinical findings and future perspectives. Int $J$ Retina Vitreous. 2017;3:14.

11. De Almeida Oliveira DC, Carvalho MOS, do Nascimento VML, et al Sickle cell disease retinopathy: characterization among pediatric and teenage patients from northeastern Brazil. Rev Bras Hematol Hemoter. 2014;36(5):340-344.

12. McLeod D. A chronic grey matter penumbra, lateral microvascular intussusception and venous peduncular avulsion underlie diabetic vitreous haemorrhage. Br J Ophthalmol. 2007;91(5):677-689.

13. Bishop PN. The role of extracellular matrix in retinal vascular development and preretinal neovascularization. Exp Eye Res. 2015;133:30-36.

14. Tomi A, Marin I. Angiofluorographic aspects in age-related macular degeneration. J Med Life. 2014;7(4):4-17.

15. Englander M, Xu D, Kaiser PK. Part 6: Retina and Vitreus. Section 2 Ancillary tests, Optical coherence tomography. In: Myron Yanoff, Jay S Duker, editors. 4th edn. Ophthalmology. Netherlands. Elsevier Saunders; 2014:454.

16. Jia Y, Bailey ST, Wilson DJ, et al. Quantitative optical coherence tomography angiography of choroidal neovascularization in age-related macular degeneration. Ophthalmology. 2014;121(7):1435-1444.

17. Kuehlewein L, Bansal M, Lenis TL, et al. Optical Coherence Tomography Angiography of Type 1 Neovascularization in Age-Related Macular Degeneration. Am J Ophthalmol. 2015;160(4):739-748.

18. Torrecillas-Picazo R, Cerdà-Ibáñez M, Almor Palacios I, et al. Analysis and follow-up of type 1 choroidal neovascularisation with optical coherence tomography-angiography after antiangiogenic treatment. Arch Soc Esp Oftalmol. 2017;S0365-6691(17):30007-30012.

19. Iuliano L. Optical coherence tomography angiography of choroidal neovascularisation. European Ophthalmic Review. 2016;10(1):29-30. 